\title{
Sistematização da Assistência de Enfermagem (SAE) na atenção primária à saúde
}

\author{
Nursing Care Systematization (SAE) in primary health
}

Sistematización de la Asistencia de Enfermería (SAE) en la atención primaria de salud

Karina Morais Wanzeler ${ }^{1 *}$, Luzia Beatriz Rodrigues Bastos ${ }^{1}$, Alexandre Barbosa da Cruz ${ }^{1}$, Nicélia Pereira da Silva ${ }^{1}$, Severa Pereira Carneiro Souza ${ }^{1}$, Diniz Antonio de Sena Bastos ${ }^{2}$, Priscila de Nazaré Quaresma Pinheiro ${ }^{3}$, Fernanda Cristina Costa Souza Costa ${ }^{1}$, Thayane Aparecida Souza Honorato $^{1}$, Gabriela Nunes Pinheiro ${ }^{1}$, Andréa da Silva Pereira Amaral ${ }^{1}$, Marciléia dos Santos Vinhas $^{1}$, Ingrid Aparecida Rodrigues Vieira ${ }^{1}$, Leane Dos Reis Costa ${ }^{1}$, Samara da Silva Barbosa ${ }^{1}$.

\section{RESUMO}

Objetivo: Analisar os fatores que interferem na implementação da Sistematização da Assistência de Enfermagem e Processo de Enfermagem, nas práticas de enfermagem na atenção primária à saúde. Método: Trata-se de estudo com abordagem qualitativa, realizada com oito (8) enfermeiros, através de entrevista semiestruturada, ocorrida nos meses de setembro e outubro de 2018, em unidades de saúde. Resultados: Da análise dos dados emergiram as categorias: Formação acadêmica na SAE; Dificuldades na aplicabilidade da SAE; Obrigatoriedade e Importância da SAE na consulta de enfermagem. Há necessidade de reavaliação dos componentes curriculares dos cursos de graduação em enfermagem e dos métodos de ensino, no sentido de promover planejamentos orientados ao destaque da SAE na atenção primária, bem como a superação de dificuldades enfrentadas pelos profissionais de enfermagem em sua aplicabilidade. Conclusão: É importante a preocupação com a formação dos futuros profissionais de enfermagem, considerando que a consolidação e defesa da ciência de enfermagem se estabelecerá concretamente a partir desta e das próximas gerações de enfermeiros.

Palavras-chave: Cuidados de Enfermagem, Processo de Enfermagem, Atenção primária à saúde.

\begin{abstract}
Objective: To analyze the factors that interfere in the implementation of the nursing care systematization and Nursing Process, in nursing practices in primary health care. Method: It is a study with a qualitative approach, performed with eight (8) nurses, through a semi-structured interview, held in September and October of 2018, in health units. Results: From the analysis of the data emerged the categories: Academic formation in SAE; Difficulties in the applicability of SAE; Obligation and Importance of SAE in nursing consultation. There is a need for a reassessment of the curricular components of undergraduate nursing courses and teaching methods in order to promote planning aimed at highlighting SAE in primary care, as well as overcoming the difficulties faced by nursing professionals in their applicability. Conclusion: It is important the concern with the training of future nursing professionals, considering that the consolidation and defense of nursing science will be established concretely from this and the next generations of nurses.
\end{abstract}

Keywords: Nursing Care, Nursing Process, Primary health care.

1 Universidade da Amazônia (UNAMA), Belém-Pará. *E-mail: karinamwanzeler@gmail.com

2 Universidade do Estado do Pará (UEPA), Belém-Pará.

${ }^{3}$ Secretaria Municipal de Saúde de Belém (SESMA), Belém-Pará. 


\section{RESUMEN}

Objetivo: Analizar los factores que interfieren en la implementación de la asistencia de enfermería y Proceso de Enfermería en las prácticas de enfermería en la atención primaria de salud. Método: Es un estudio con enfoque cualitativo, realizado con ocho (8) enfermeras, a través de una entrevista semiestructurada, realizada en septiembre y octubre de 2018, en unidades de salud. Resultados: Del análisis de los datos surgieron las categorías: Formación académica en SAE; Dificultades en la aplicabilidad de SAE; Obligación e Importancia de SAE en consulta de enfermería. Es necesario reevaluar los componentes curriculares de los cursos de pregrado de enfermería y los métodos de enseñanza para promover la planificación orientada a resaltar las SAE en la atención primaria, así como superar las dificultades que enfrentan los profesionales de enfermería en su aplicabilidad. Conclusión: Es importante la preocupación con la capacitación de futuros profesionales de enfermería, considerando que la consolidación y la defensa de la ciencia de enfermería se establecerán concretamente a partir de esta y las próximas generaciones de enfermeras.

Palabras clave: Atención de enfermería, Proceso de enfermería, Atención primaria de salud.

\section{INTRODUÇÃO}

A Sistematização da Assistência de Enfermagem (SAE), é uma ferramenta científica que confere maior segurança e qualidade da assistência aos pacientes, e maior autonomia aos profissionais de enfermagem. Na prática se constitui na aplicabilidade da ciência de enfermagem (SANTANA JCB, et al., 2013).

Ao fazer a SAE, o enfermeiro organiza o processo de trabalho é organizar um processo de trabalho voltado à equipe, não estando vinculado somente ao atendimento individual dos enfermeiros, mas a todos os membros da equipe de enfermagem, uma vez que possuem atribuições pertinentes que thes são delegadas pela lei do exercício profissional (GONÇALVES RC, 2015).

O enfermeiro vivencia um desafio na edificação e compilação do conhecimento sobre o qual se fundamenta sua prática gerencial e assistencial. Assim, a SAE vem para somar e conformar o planejamento, a execução, o controle e a avaliação das ações de cuidados direto e indireto ao indivíduo e suas famílias (SOARES MI, et al., 2015).

O Processo de Enfermagem (PE) operacionaliza a SAE, enquanto instrumento metodológico que orienta o cuidado profissional de enfermagem e a documentação da prática, composta por cinco etapas, a investigação, diagnóstico de enfermagem, planejamento, implementação da assistência e a avaliação, etapas que se inter-relacionam de modo interdependentes e recorrentes (COFEN, 2009).

Nesse contexto, a assistência de enfermagem pautada no Processo de Enfermagem, tem potencial para favorecer a integralidade e a promoção da saúde necessárias para superar as dificuldades da atenção primária à saúde e sua articulação com outros níveis de atenção (média e alta complexidade), seguindo uma ordem crescente de complexidade da assistência, garantindo que cada indivíduo seja atendido no nível que necessita, alcançando de forma eficiente a saúde individual e coletiva (SILVA JP, GARANHANI M, MENEZES MHDG, 2014).

A experiência cotidiana tem demonstrado que há grandes dificuldades na implementação do Processo de Enfermagem (PE), na atenção primária à saúde (APS). Em razão disso, o estudo proposto apresenta como objetivo, analisar os fatores que interferem na sistematização da assistência de enfermagem pela implementação do processo de enfermagem, nas práticas de enfermagem na atenção primária à saúde (APS).

\section{MÉTODOS}

Estudo com abordagem qualitativa, realizado com oito (8) enfermeiros (as), efetivos e temporários, atuantes em programas de saúde, tais como: Programa Saúde da Criança, Programa de Atenção ao 
Hipertenso e Diabético (Hiperdia), Polo do Idoso, Programa de Controle e Combate à Tuberculose e Hanseníase e Programa do Pré-Natal, em unidades municipais de saúde de Belém/PA.

A coleta de dados ocorreu, durante os meses de setembro e outubro de 2018 , nos turnos matutino e vespertino, pautada em entrevista semiestruturada, que aconteceram na própria unidade de saúde sempre após o expediente de trabalho dos profissionais. Para análise dos dados, se utilizou a técnica de análise de Conteúdo (BARDIN L, 2011), que possibilitou organizar o conjunto das falas emitidas pelos enfermeiros e descobrir os núcleos de sentido que compunham a comunicação cuja presença ou frequência puderam revelar aspectos significativos.

A análise dos dados ocorreu através dos passos: leitura dos artigos; escolha e demarcação de segmentos do texto para análise; formulação do objetivo; exploração do material com a definição de categorias; análise crítica com a interpretação das mensagens. Como toda pesquisa envolve riscos, os pesquisadores garantiram o sigilo das informações coletadas e armazenadas, assegurando o anonimato com a utilização do código ENT. (entrevistado), seguido do número de ordem de cada pesquisado, dificultando assim qualquer identificação.

Com o intuito de obedecer às diretrizes que regulamentam os estudos que envolvem seres humanos, foi apresentando o Termo de Consentimento Livre e Esclarecido (TCLE), com dados referentes ao caráter voluntário do estudo e possibilidade de desistência a qualquer momento, sem que haja qualquer retaliação, impedimento ou discriminação ao mesmo. O estudo ocorreu após a aprovação no Comitê de Ética em Pesquisa, da Universidade da Amazônia/UNAMA, vinculada à Plataforma Brasil, atendendo as exigências do TCLE, cumprindo os requisitos da Resolução 466/12 do Conselho Nacional de Saúde, sob o número CAAE 90715718.3.0000.5173.

\section{RESULTADOS E DISCUSSÃO}

As entrevistas foram realizadas com oito (8) enfermeiros, sendo sete (7) do sexo feminino, com idade média de 36 anos. Os pesquisados tinham aproximadamente 12 anos de formados, com tempo de trabalho na atenção primária de 5 anos. No que diz respeito à titulação, seis (6) entrevistados possuíam especialização e o restante (2), a graduação em enfermagem.

Quadro 1 - Caracterização dos enfermeiros entrevistados nas Unidades Municipais de Saúde de Belém/PA, 2018.

\begin{tabular}{|l|l|}
\hline Entrevistados & N \\
\hline Sexo & 7 \\
\hline Feminino & 1 \\
\hline Masculino & \multicolumn{2}{|l|}{} \\
\hline Idade & 36 Anos \\
\hline Média das Idades & 12 anos \\
\hline Escolaridade & 5 anos \\
\hline Ano de Formação & \multicolumn{2}{|l|}{} \\
\hline Tempo de Trabalho & 6 \\
\hline Titulação & 2 \\
\hline Especialização & \\
\hline Graduação &
\end{tabular}

Fonte: Wanzeler KM, Bastos LBR, Cruz AB, et.al, 2018. 
A partir dos relatos, foram originadas quatro categorias de análise: Formação acadêmica na SAE; Dificuldade na aplicabilidade da SAE na APS; Obrigatoriedade na aplicabilidade da SAE; e Importância da SAE na consulta de enfermagem.

\section{1aㅡ Categoria: Formacão Acadêmica na SAE/ Processo de Enfermagem.}

Nesta categoria temática, a maioria dos entrevistados relataram que o ensino da SAE nas graduações foi pouco explorado, principalmente nos semestres iniciais, conforme demonstrado a seguir:

Sim! Mas foi muito pouco abordado pois estavam iniciando a implantação da SAE nas universidades (ENT.1).

Sim! A disciplina SAE era abordada no 1ํsemestre com teoria e prática, se aplicando todos os processos desde a anamnese até ao diagnóstico (ENT.4).

Sim foi muito pouco, pois estava começando então nesse tempo não tínhamos muita experiência com a disciplina (ENT.5).

É importante ressaltar que a SAE é uma forma de possibilitar um trabalho sistemático pela implementação do PE e suas etapas, proporcionando aos usuários, uma abordagem completa de seu estado de saúde, com descobertas ágeis nos diagnósticos e no tratamento dos problemas de saúde. Torna-se importante como mecanismo de valorização e autonomia da Enfermagem na APS, possibilitando aos enfermeiros um diferencial em suas consultas (CHAVES RRG, et al., 2016).

Observa-se que, apesar da tentativa de introduzir, nas séries iniciais do curso, elementos que compõem a SAE, os acadêmicos não conseguem compreendê-los, o que revela falta de explicitação sobre a relação do PE com a anamnese e exame físico. Esta dificuldade repercute, inclusive, na capacidade de apreender 0 tema e na formação do profissional Enfermeiro (PORFÍRIO JS, GARANHANI ML, MARIS PERES A, 2015).

O tempo de formação contribui na implantação e utilização do PE e no atendimento prestado ao paciente. Os profissionais que atuam na graduação e nos cursos técnicos nas instituições de saúde brasileiras, devem possuir conhecimento prévio da implantação do processo de sistematização de enfermagem (SILVA JP, GARANHANI M, MENEZES MHDG, 2014).

Como a prática dos profissionais reflete, sobremaneira, sua formação, as Instituições de Ensino Superior têm grande responsabilidade em propiciar o desenvolvimento de habilidades, a seus acadêmicos, para a realização da SAE (VENTURINI DA, MATSUDA LM, WAIDMAN MAP, 2009).

Faz-se necessário que na graduação e nos cursos técnicos o PE seja ensinado de maneira aprofundada, devendo ser incorporada nas atitudes clínicas do enfermeiro e equipe, em suas rotinas de trabalho. $O$ processo de enfermagem desenvolve o raciocínio e julgamento clínico nas decisões diagnósticas e de intervenção de enfermagem (GOES JF, FERREIRA RMC, 2016).

O ensino da SAE e PE adotados nas instituições de ensino superior precisam ser reavaliados durante o processo de planejamento das ações pedagógicas das disciplinas e de todas as atividades que norteiam o curso de graduação em enfermagem. O professor necessita encontrar estratégicas e técnicas de modo a favorecer o ensino de forma mais dinâmica e criativa, fazendo uso das metodologias ativas, dentre elas, simulações realísticas, vídeos, textos, dentre outros (MEDEIROS AL, SANTOS SR, CABRAL RWL, 2013).

É importante a preocupação com a formação dos futuros profissionais de enfermagem, considerando que o processo de consolidação e defesa da ciência de enfermagem se estabelecerá concretamente a partir desta e das próximas gerações de enfermeiros (as).

A articulação teórico-prática, advinda da formação profissional do enfermeiro, é importante para a aquisição das competências necessárias à realização da consulta de Enfermagem, que possui valor bastante significativo para dar resolubilidade às questões apresentadas pelos pacientes, permitindo atendê-los de maneira holística e integral (OLIVEIRA SKP, et al., 2012). 


\section{2a Categoria: Dificuldades na Aplicabilidade da SAE na APS}

A maioria dos pesquisados relataram dificuldades em aplicar o PE no cotidiano profissional, argumentando falta de tempo; sobrecarga de trabalho em relação à grande demanda de usuários; e prolongamento na duração das consultas de enfermagem, conforme demonstrado a seguir:

Sim. Pois para ser bem executada necessita de um tempo com o paciente para poder realizar cautelosamente à $S A E$, mas devido ao grande número de paciente não se tem tempo (ENT.5).

Sim. A falta de tempo para a assistir com qualidade o paciente, devido ao grande número de paciente para serem atendidos (ENT. 6).

Sim! Devido ao grande número de programas nas unidades a serem prestados e ainda ao grande número de paciente diário atendidos (ENT.8).

As dificuldades na implantação da SAE podem ser atribuídas a vários fatores os mais encontrados foram fatores pessoais, profissionais e organizacionais. Para superar essas dificuldades e necessário que os profissionais responsáveis busquem referência teóricas para assim conseguir alcançar os objetivos propostos na assistência (SOARES VS, et al., 2013).

Foram apontados como entraves na implementação do PE um número reduzido de profissionais, falta de credibilidade dos técnicos de enfermagem, desconhecimento, questões políticas, sobrecarga de trabalho, falta de tempo, falta de vontade dos gestores em implantar a SAE, falta de motivação profissional, dificuldades de relacionar a teoria coma prática (MEDEIROS JBP, SILVA EG, 2018).

Percebe-se que a deficiência nos registros do PE, o torna informal, incompleta e inoperante, dificultando sua implementação. Muitos são os fatores que interferem na sua aplicabilidade, dentre eles, os organizacionais, e o próprio profissional em relação às suas atitudes, crenças, valores e habilidades técnicas Atualmente sua implementação é considerada um desafio, tanto para o gerenciamento da assistência quanto para o enfermeiro, pois exige empenho e criatividade para sua execução (SOARES, et al., 2015).

Estudos apontam alguns problemas que são enfrentados pelos enfermeiros quando da implementação da SAE, como o desconhecimento em relação ao processo de trabalho da SAE, dificuldades com o próprio ensino da sistematização, falta de capacitação para o desenvolvimento das atividades na atenção primária a saúde, dentre outras (SOARES VS, et al., 2013).

Ressalta-se que o investimento em educação continuada da equipe de enfermagem, permitirá fazer o diferencial em termos de conhecimento teórico-prático pautado na SAE, para a realização de uma assistência de enfermagem qualificada.

\section{Categoria: Obrigatoriedade da SAE}

Quando questionados sobre a exigência de obrigatoriedade da SAE na atenção primária, quanto ao cumprimento da legislação do Conselho Federal de Enfermagem/2009, demonstraram falta de exigência nos locais de trabalho; destaque da aplicabilidade da SAE no campo hospitalar; desconhecimento dos gerentes das unidades de saúde; e falta de protocolos que sustentem sua aplicabilidade, como demonstrado a seguir:

Não! Não costumo colocar em prática a SAE, pois nos locais onde eu trabalhei não exigia e não tinha um protocolo que determinasse (ENT.2).

Não! Pois eu trabalho só aqui. No hospital, era padronizado tinha formulário. Só tinha que ir marcando as respostas (ENT.6).

Não, até porque a gerente nem sabe o que é SAE (ENT.7).

As informações declaradas pelos enfermeiros divergem da normativa do COFEN/358/2009, que legaliza a SAE, enfatizando sua obrigatoriedade e necessidade de sua aplicabilidade na prática cotidiana da enfermagem em diferentes cenários de trabalho, preconizada como uma atividade privativa do enfermeiro, 
baseada em estratégias científicas planejadas para a identificação das diversas situações do binômio saúde/doença (SOARES MI, et al., 2015).

A referida resolução COFEN/358/2009 estabelece no artigo 1을

"O Processo de Enfermagem deve ser realizado de modo deliberado e sistemático em todos os ambientes públicos ou privados, em que ocorre o cuidado profissional de Enfermagem".

A aplicabilidade da SAE é realizada por meio do Processo de Enfermagem (PE), que possibilita ao profissional identificar, descrever, compreender os planos de cuidado e traçar as intervenções de enfermagem, proporcionando uma melhor qualidade da assistência, aumentando a satisfação e crescimento da enfermagem, permitindo aplicar os conhecimentos teóricos na prática, fortalecendo-a enquanto ciência, tornando-a mais precisa e eficiente da qualidade, conferindo maior segurança aos pacientes e maior autonomia aos profissionais (VENTURINI DA, MATSUDA LM, WAIDMAN MAP, 2009).

Embora seja um processo dinâmico e nem sempre linear, para fins didáticos e permitir melhor compreensão, o PE é organizado em cinco etapas subsequentes e inter-relacionadas: histórico; diagnóstico; planejamento; implementação e avaliação ou evolução de Enfermagem (COFEN, 2009).

O Histórico de enfermagem corresponde à coleta de informações do sujeito, família ou coletividade de forma ordenada, organizada e longitudinal, cujas informações são significativas para o raciocínio clínico do enfermeiro, por possibilitar a identificação de problemas e potencialidades (SILVA VM, et al., 2014).

O diagnóstico de enfermagem, pauta-se no desvelamento das informações coletadas no histórico, bem como seu agrupamento, emergindo daí a tomada de decisão sobre as necessidades do paciente e família, fazendo uso de conceitos diagnósticos de enfermagem (BEVILACQUA P, MELO CM, BARLETTO M, 2017).

A etapa relativa ao planejamento de enfermagem visa a escolha dos resultados que se deseja alcançar $\mathrm{e}$ as respectivas ações de enfermagem para que os resultados ocorram em tempo hábil. Isto baseado nas respostas da pessoa, família ou coletividade humana em um dado momento do processo de saúde/doença ou processo de vida, identificadas nas etapas do Histórico e Diagnóstico de Enfermagem (COFEN, 2009).

A implementação é a realização das ações ou intervenções propostas na fase de planejamento; e a Avaliação ou Evolução de Enfermagem corresponde à avaliação global da prescrição de enfermagem implementada, considerando as repercussões da assistência na condição do paciente, família ou comunidade. Corresponde ao processo dinâmico apresentado pelo paciente (BEVILACQUA P, MELO CM, BARLETTO M, 2017).

\section{Categoria: Importância da SAE na consulta de enfermagem}

No que se diz respeito à utilização da SAE na consulta de enfermagem aplicando o PE e suas etapas, a maioria dos pesquisados, enfatizaram sua importância no intuito de promover a melhoria do atendimento aos usuários, conforme referido:

Sim! Pois pode trazer inúmeros benefícios para o paciente mediante atendimento de forma mais humana, eficiente a técnica (ENT.2).

Sim! Pois promove uma assistência diferenciada e sistematizada (ENT.5).

Sim, o trabalho fica mais organizado e isso pode repercutir na melhora da assistência (ENT.6).

Nesse contexto, os pesquisados demonstraram que acreditam na melhoria do atendimento ao usuário quando a SAE é utilizada como uma metodologia de sistematização das ações e organização do trabalho. No entanto, grande parte não a utiliza.

Os enfermeiros reconhecem que o foco da assistência deve ser o usuário, com enfoque em suas necessidades, a questão está em torná-la uma realidade. 
A SAE subsidia ações que possam contribuir para a promoção, prevenção, recuperação e reabilitação da saúde do indivíduo, a partir de etapas através do Processo de Enfermagem (PE), com o planejamento, organização, execução e avaliação, guiadas por uma teoria de enfermagem (COFEN, 2009).

$\mathrm{Na}$ consulta de Enfermagem, o enfermeiro deve se preocupar com a implementação de práticas que ofereçam condições seguras e de qualidade para o desempenho de suas atividades. Os usuários procuram a consulta de Enfermagem para orientação da prática do autocuidado, para adquirir informações acerca da doença e do tratamento, visto que uma das ações do enfermeiro é orientar o paciente conforme suas necessidades para promoção e recuperação da saúde (OLIVEIRA SKP, et al., 2012).

\section{CONSIDERAÇÕES FINAIS}

A atenção primária a saúde é a porta de entrada do Sistema Único de Saúde, para acesso aos programas de saúde disponibilizados em nível ministerial para a população usuária do sistema público de saúde. Nesse cenário a enfermagem está presente em todos os programas ofertados pelas unidades de atenção primária. Destaca-se a importância da implementação da SAE, como um mecanismo de valorização, autonomia e de práticas de enfermagem de qualidade. Oferece ao enfermeiro uma forma de cuidar diferenciada possibilitando a detecção precoce de problemas, possibilitando um trabalho organizado com melhores alternativas de respostas aos problemas de saúde dos usuários.

\section{REFERÊNCIAS}

1. BARDIN L. Análise de Conteúdo. Trad. Pinheiro, LARA. São Paulo: Edições 70; 2011.

2. BEVILACQUA P, MELO CM, BARLETTO M. Educação Permanente em Vigilância em Saúde: formação política e reorientação de práticas em serviços. 2017:78.

3. CONSELHO FEDERAL DE ENFERMAGEM. Resolução COFEN no 358/2009. Brasília (DF): Conselho Federal de Enfermagem (COFEN), 2009. Disponível em www.cofen.gov.br: Acesso em 25.mai. 2018.

4. CHAVES RRG, et al. Sistematização da Assistência de Enfermagem: visão geral dos enfermeiros. Rev. enferm. UFPE online. 2016, 10(4):1280-85.

5. GOES JF, FERREIRA RMC. Dificuldades da equipe de enfermagem na implantação da sistematização da assistência de enfermagem no centro cirúrgico de Campo Grande - MS. Revista Convibra. 2016.

6. GONÇALVES RC. Manual para técnicos/auxiliares de enfermagem. 2015.

7. MEDEIROS JBP, SILVA EG. Hemodinâmica: implementação de assistência de enfermagem durante a hemodiálise. Revista de Divulgação Científica Sena Aires. 2018, 7(3):182-91.

8. MEDEIROS AL, SANTOS SR, CABRAL RWL. Desvelando dificuldades operacionais na sistematização da assistência de enfermagem através da Grounded Theory. Revista Eletrônica de Enfermagem, Goiânia. 2013, 15(1):44-3.

9. OLIVEIRA SKP, et al. Temas abordados na consulta de enfermagem: revisão integrativa da literatura. Revista Brasileira de Enfermagem.2012, 65(1).

10. PORFÍRIO JS, GARANHANI ML, MARIS PERES A. Sistematização da assistência de enfermagem na graduação: um olhar sob o pensamento complexo. Revista Latino-Americana de Enfermagem. 2015, 23(1).

11. SANTANA JCB, et al. Percepção dos enfermeiros acerca da Sistematização da Assistência de Enfermagem na atenção básica de Belo Horizonte. Enfermagem Revista. 2013, 16(1):4-17.

12. SILVA VM. et al. Morbidity in users of Family Health teams in the northeast of Minas Gerais based on the International Classification of Primary Care. Revista Brasileira de Epidemiologia. 2014, 17:954-67.

13. SILVA JP, GARANHANI M, MENEZES MHDG. Sistematização da assistência de enfermagem e o pensamento complexo na formação do enfermeiro: análise documental. Revista Gaúcha de Enfermagem.2014, 35(2):128-34.

14. SOARES VS, et al. Utilização do processo de enfermagem e as dificuldades encontradas por enfermeiros. Cogitare Enferm. 2013, 18(2):351-7.

15. SOARES MI, et al. Sistematização da assistência de enfermagem: facilidades e desafios do enfermeiro na gerência da assistência. Escola Anna Nery Revista de Enfermagem. 2015, 19(1):47-53.

16. VENTURINI DA, MATSUDA LM, WAIDMAN MAP. Produção científica brasileira sobre sistematização da assistência de enfermagem. Ciência, Cuidado e Saúde. 2009, 8(4):707-15. 